\title{
Regional Distribution of Choroidal Thickness and Diurnal Variation in Choroidal Thickness and Axial Length in Young Adults
}

\author{
Muteb Alanazi $\mathbb{D}^{1,2}$ \\ Patrick Caroline ${ }^{2}$ \\ Amane Alshamrani (iD) ${ }^{2}$ \\ Turki Alanazi ${ }^{3}$ \\ Maria Liu ${ }^{4}$ \\ 'Optometry Department, College of \\ Applied Medical Sciences, King Saud \\ University, Riyadh, Saudi Arabia; ${ }^{2}$ College \\ of Optometry, Pacific University, Forest \\ Grove, OR, USA; ${ }^{3}$ School of Engineering \\ and Applied Science, The George \\ Washington University, Washington, DC, \\ USA; ${ }^{4}$ UC Berkeley School of \\ Optometry, Berkeley, CA, USA
}

Purpose: To examine the regional distribution of choroidal thickness (ChT) and the diurnal variation in ChT and axial length (AL) over a wide range of myopic refractive error.

Methods: ChT was measured in thirty-four healthy young adults (age mean \pm SD: $25.2 \pm$ 2.8, range: 18-35 years) using spectral-domain optical coherence tomography and AL using an IOL-Master 500. Participants were divided into three refractive groups: emmetropes, myopes, and high myopes. We evaluated ChT in macular (foveal, parafovea, and perifovea) and peripheral regions (6-mm from the foveal pit) in four quadrants (superior, temporal, inferior, and nasal). To assess the diurnal variation, three measurement sessions of ChT and $\mathrm{AL}$ were taken at $8 \mathrm{AM}, 12 \mathrm{PM}, 4 \mathrm{PM}$.

Results: ChT thins progressively towards the periphery. Superior and nasal quadrants exhibited the thickest $(277 \pm 73 \mu \mathrm{m})$ and thinnest $(218 \pm 89 \mu \mathrm{m})$ choroid, respectively. Higher myopic eyes showed an overall thinner choroid $(237 \pm 48 \mu \mathrm{m})$ compared to myopic eyes $(264 \pm 78 \mu \mathrm{m})(\mathrm{P}<0.05)$. Higher myopes exhibited a significant choroidal thinning in all quadrants except in the temporal quadrant (all $\mathrm{p}<0.05$ ). Both ChT and AL underwent a significant diurnal variation $(\mathrm{p}<0.05)$. The $\mathrm{ChT}$ and AL diurnal variation amplitudes in higher myopes were significantly reduced (ChT: $14.6 \pm 11$, AL: $14.5 \pm 13 \mu \mathrm{m})$, compared to those in emmetropes (ChT: $21.4 \pm 15$, AL: $21.3 \pm 8.5 \mu \mathrm{m}$ ) and myopes (ChT: $19 \pm 17$, AL: $19 \pm 9.7 \mu \mathrm{m})$. Diurnal variation amplitude in ChT did not differ significantly across quadrants and choroidal eccentricity regions $(\mathrm{p}>0.05)$.

Conclusion: ChT distribution varies based on quadrant and eccentricity; superior choroid exhibited the thickest, and nasal showed the thinnest choroid. Higher myopes experience a reduced diurnal variation in $\mathrm{ChT}$ and $\mathrm{AL}$.

Keywords: choroid, diurnal variation, myopia, optical coherence tomography

\section{Introduction}

The human body goes under daily intrinsic variations in physiological parameters called the circadian rhythm. ${ }^{1}$ As a part of the human body's physiological processes, several ocular structures showed a consistent physiologic diurnal variation in intraocular pressure, corneal thickness, axial length (AL), and choroidal thickness (ChT). ${ }^{2}$

Diurnal variation in AL was first observed in chicks then later demonstrated in humans. ${ }^{3}$ Most studies that investigated AL rhythms in humans showed that the AL is shortest during the night and longest during the day. It is well known the choroid also undergoes diurnal fluctuations in thickness (increasing during the night and
Correspondence: Muteb Alanazi Tel +9665651227716

Email mkalanazi@ksu.edu.sa 
decreasing during the day) in animal models and in humans. ${ }^{2}$ Previous research revealed that the amplitude of ChT diurnal variation is correlated with AL. Also, AL and ChT diurnal variations are in antiphase of one another. $^{2,4,5}$

The choroid plays a significant role in the pathogenesis of a variety of ocular diseases. Increased ChT was reported to be associated in patients with chronic central serous choroioretinopathy $^{6}$ and Vogt-Koyanagi-Harada disease. ${ }^{7}$ Additionally, a significant thinning of the choroid was observed with several conditions such as pathologic myopia, ${ }^{8}$ age-related macular degeneration, ${ }^{9}$ glaucoma, ${ }^{10}$ and diabetic retinopathy. ${ }^{11}$ Evidence also suggests that the choroid communicates information from the retina to the sclera, acting as a regulator of scleral growth. ${ }^{12}$

Technological advances in optical coherence topographies have made assessing the diurnal variation in ChT in humans achievable. Numerous recent clinical studies suggest that the choroid and the changes in ChT could be an important biomarker in evaluating ocular disease; however, the changes in the $\mathrm{ChT}$ are not restricted to macular regions. Most recently published studies investigating the diurnal variation of $\mathrm{ChT}$ had been limited to the subfoveal choroidal region. Regarding the distribution of wide-field ChT, Rasheed et al measured ChT along the vertical and horizontal meridians and reported a significantly reduced thickness at peripheral points compared to the subfoveal region in all quadrants. ${ }^{13}$ Similar results were described by Hoseini-Yazdi et $\mathrm{al}^{14}$ and others. ${ }^{15,16}$ The findings have shown that ChT varies with choroidal quadrant (exhibits thickest in superior quadrant and thinnest in nasal) and eccentricity (where it progressively thins towards the periphery).

The normal physiologic diurnal fluctuation in ocular components including ChT may be critical in maintaining normal ocular growth and refractive development. Findings from previous research in animals ${ }^{17}$ and humans ${ }^{18}$ indicated that diurnal variations in AL and ChT might be altered during refractive error development.

ChT is negatively correlated with refractive error. Eyes with high myopia exhibit thin choroid in adults ${ }^{4,14,16}$ and children. ${ }^{19}$ In this study, we sought to further investigate the amplitude and the pattern of the physiologic diurnal fluctuation in $\mathrm{ChT}$ and $\mathrm{AL}$ in eyes over a wide range of myopic refractive error across a wide field. Highly myopic eyes were reported to have a thinner choroid, which may demonstrate a different pattern and a restricted physiologic diurnal variation in $\mathrm{ChT}$ and $\mathrm{AL}$ in comparison to emmetropic and myopic eyes.

\section{Methods}

\section{Subjects}

This study followed the tenets of the Declaration of Helsinki, and approval was obtained from the Institutional Review Board (IRB) before the commencement of the study. All participants gave their informed written consent prior to study participation after being informed about the nature and possible consequences of participating in the study. Subjects were screened before enrolment and found to be in good ocular health and free from ocular disease.

Only adult participants between the age of 18 to 35 years were recruited. Thirty-four adult subjects participated in this study. All participants underwent initial screening, which included subjective refraction and visual acuity. All subjects had normal best-corrected logarithmic minimum angle of resolution ( $\log M A R)$ visual acuity of 0.1 or better. Subjects with best-corrected LogMAR worse than 0.1 were excluded. The participants were divided into three refractive groups: emmetropes (spherical equivalent refraction between +0.50 and $-0.49 \mathrm{D})$, myopes $(\leq-0.50$ and $>-6.00 \mathrm{D})$, and high myopes $(\leq-6.00 \mathrm{D})$. No retinal diseases nor systemic diseases have been reported from any of the subjects. Subjects who suffered from retinal diseases and/or systemic conditions such as diabetes and hypertension were excluded from the study. Subjects who wore contact lens were asked to discontinue wearing contact lens the night prior to the day of the study. Since alcohol, ${ }^{20}$ caffeine, ${ }^{21}$ and cigarette smoking ${ }^{22}$ were found to have an influence on ChT, all subjects were instructed not to drink alcoholic and caffeinated beverages and do not smoke cigarettes the night before and on the day of the study.

\section{Measurements and Data Collection Procedures \\ Choroidal Thickness (ChT)}

Both eyes of each participant were examined using Optovue optical coherence tomography (Optovue Inc., Fremont, CA). Five high-resolution (496 pixels per A-scan, 1024 A-scans per B-scan) 12-mm single scans across the horizontal meridian and another five single scans were taken across the vertical meridian. All scans were centered on the fovea. Each horizontal and vertical 
line scan is a result of 60 averaged B-scans. This scanning protocol covers an approximate retinal angle of $47^{\circ} \times 47^{\circ}$ horizontally and vertically for an AL of $24 \mathrm{~mm}$ (Figure 1).

The ChT was quantified automatically using software developed by Alonso-Caneiro et al 2013 and Read et al 2015. Studies have shown there are differences in ChT at each location (superior, inferior, nasal, and temporal), ${ }^{14}$ where the superior choroid exhibited the thickest thickness, and the nasal choroid showed the thinnest. Due to the observed significant variation in ChT based on the location, we recorded and analyzed the data for each location separately. In each quadrant, the ChT measurements were divided into five anatomical areas (fovea, parafovea, perifovea, near-periphery, and periphery (Figure 1). The
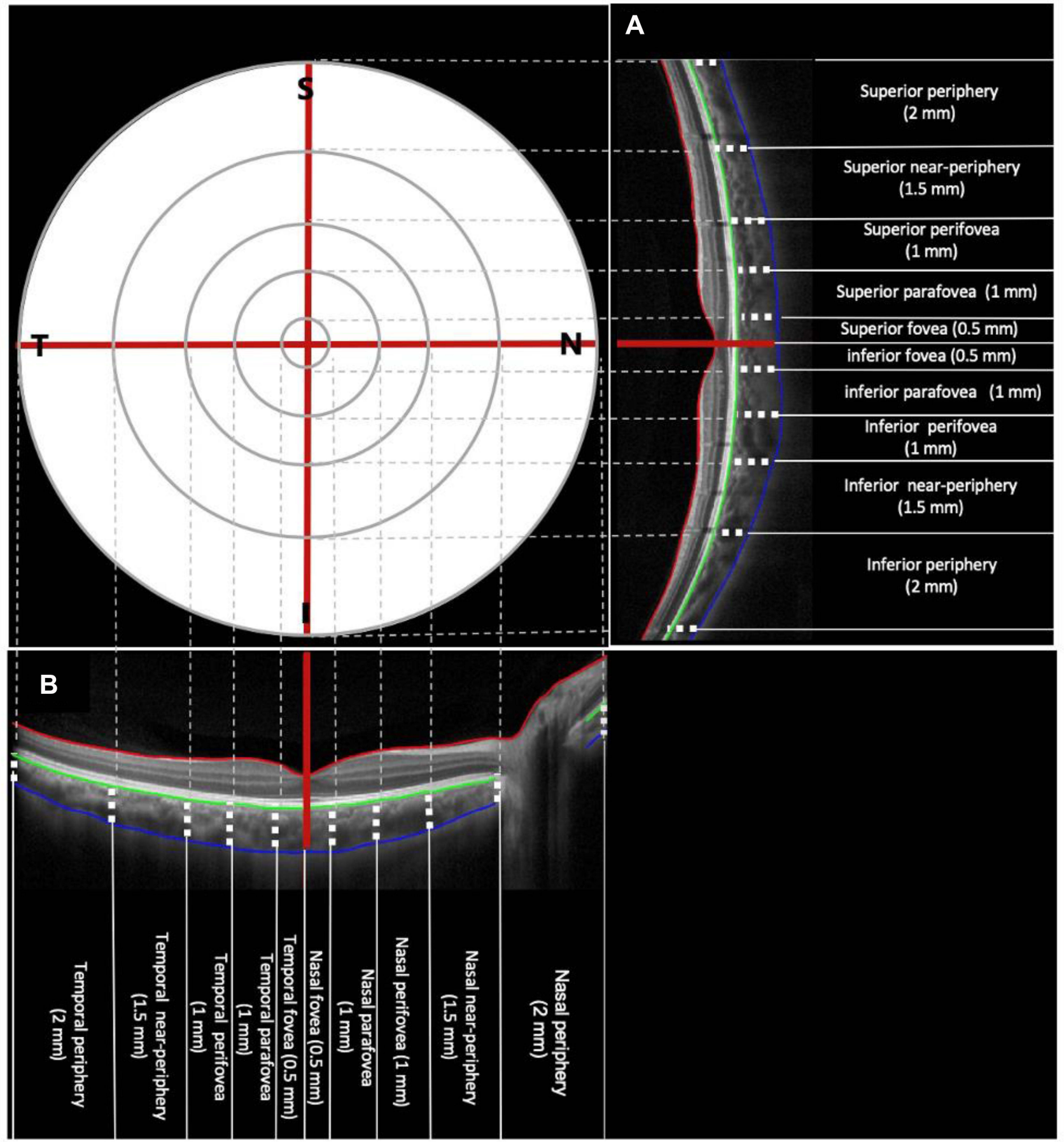

Figure I Vertical (A) and horizontal (B) single line image obtained with optical coherence tomography (OCT) instrument, the foveal pit as manually marked in the customwritten software (straight red line in ( $\mathbf{A}$ and $\mathbf{B}$ ), the anterior (green line in ( $\mathbf{A}$ and $\mathbf{B}$ ) and posterior (blue line in $(\mathbf{A}$ and $\mathbf{B})$ are the boundaries of the choroid. 
software contained image contrast enhancement that improves the visualization of the choroidoscleral junction. $^{23}$

Other factors have been reported to have a potential impact on ChT and AL measurements, such as physical activity, $^{24}$ near work, ${ }^{25}$ and the ambient light. ${ }^{26}$ To minimize the impact from these factors, the subject spent 15 minutes in a room with low photopic ambient light levels while looking at a distant target placed at 3 meters prior to taking measurements. Following the OCT scans segmentation, the transverse scaling of each scan was corrected based on the participant's axial length to account for ocular magnification using a method previously described by Read et al. ${ }^{27}$

\section{Ocular Axial Length (AL) Measurements}

A noncontact partial coherence laser interferometry (IOLMaster, Zeiss, Germany) was used to obtain the measurements of AL and anterior chamber depth. The AL measured with IOL-Master is defined as the distance from the front cornea corneal surface to the retinal pigment epithelium (RPE). Twenty repeated measurements of the AL were taken, and five readings with signal-to-noise ratio greater than seven were selected and averaged for data analysis.

\section{Data Collection Procedures}

A total of three measurement sessions were conducted at 4-hour intervals, with the first measurement taken at approximately $8 \mathrm{AM}$ and the final measurement at approximately 4 PM. Each session lasted approximately 30 minutes to complete all the measurements. In order to avoid any induced corneal epithelial disruption, only noncontact techniques were used to obtain all measurements, and no anesthetic eye drops were used.

\section{Statistical Analysis}

All the measured variables were expressed as mean with standard deviation (mean $\pm \mathrm{SD}$ ). The data of AL and ChT in all measured regions were normally distributed $(p>$ 0.05 Kolmogorov-Smirnov test for all regions). In order to examine the diurnal variation in different choroidal regions and $\mathrm{AL}$, repeated-measure analysis of variance was performed, including the within-subject factors of choroidal eccentricity (5 levels) and quadrant (4 levels), time of day (3 levels), and refractive error group (3 levels) as a between-subject factor. Pearson correlation was used to study the relationship between refractive error and diurnal variation amplitude in ChT and AL.

\section{Results}

\section{Inter-Rater Reliability: Choroidal}

\section{Thickness Measurement}

The ChT measurements were obtained using automated software developed by Alonso-Caneiro et $\mathrm{al}^{28}$ and Read et al. ${ }^{29}$ Since manual modifications may be needed on some of the OCT images to confirm the choroid borderlines, the inter-rater reliability was verified prior to the image analysis of the full dataset. Thirty random images were initially segmented by two raters (MA and AA) to determine inter-rater agreement. The inter-rater reliability for ChT was measured by the absolute agreement model of the intra-class correlation coefficient (ICC). We also performed Bland-Altman plot analyses to determine the mean difference between the measurements. Inter-rater reliability were consistent and repeatable with a ICC of 0.996 [95\% CI 0.994-0.997]. The mean difference in choroid thickness measurements between raters was $0.18 \pm 2.45$ $\mu \mathrm{m}$, with a maximum difference of $10.7 \mu \mathrm{m}$. BlandAltman plot analysis of inter-rater reliability for ChT also demonstrated optimal results.

\section{Distribution of Choroidal Thickness}

There were no statistically significant differences in any of the variables between right and left eyes $(p>0.05)$ or between female and male participants $(p>0.05)$. The mean age of the study subjects was 25.18 (range from 18 to 35 years, $\mathrm{SD} \pm 2.77$ ), with 24 females and 7 males. The mean of ChT values was 257 (range 79 to $488, \mathrm{SD} \pm 81$ ). For the purpose of additional analyses, the subjects were divided into three myopic refractive groups: emmetropes, myopes, and high myopes. Participant demographic data are listed in Table 1.

ChT significantly thinned with eccentricity $(\mathrm{p}<0.001)$. Foveal and parafoveal ChT did not differ $(p=0.99)$. The choroid progressively thinned beyond parafovea, reaching a minimum value in the periphery of $225 \mu \mathrm{m}(\mathrm{p}<0.01$ for all pairwise comparisons). ChT varied based on quadrants. The thickest was observed in the superior choroid $(277 \pm$ $73 \mu \mathrm{m})$ followed by temporal $(262 \pm 74 \mu \mathrm{m})$ and inferior $(255 \pm 80 \mu \mathrm{m})$, and thinnest in the nasal quadrant $(218 \pm$ $89 \mu \mathrm{m})(\mathrm{p}<0.05$ for all pairwise comparisons except for temporal and inferior choroid). 
Table I Participant Demographic Data and Ocular Characteristics

\begin{tabular}{|l|c|c|}
\hline & Mean \pm SD & Range \\
\hline Age (year) & $25.18 \pm 2.77$ & -35 \\
\hline Distance best corrected VA (logMAR) & $-0.06 \pm 0.08$ & $39.34-46.64$ \\
\hline Average corneal curvature (D) & $43.46 \pm 1.44$ & -0.1 \\
\hline Central spherical equivalent refractive error (D) & & $-10.54-+0.02$ \\
\hline All subjects $(n=34)$ & $-3.65 \pm 3.07$ & $-0.40-+0.02$ \\
Emmetrope $(n=6)$ & $-0.20 \pm 0.12$ & $-5.88--0.59$ \\
Myope $(n=16)$ & $-2.48 \pm 1.30$ & $-10.54--6.21$ \\
High myope $(n=12)$ & $-7.60 \pm 1.58$ & $22.49-27.20$ \\
\hline Axial length $(m m)$ & & $22.49-24.66$ \\
\hline All subjects $(n=34)$ & $24.85 \pm 1.18$ & $22.97-25.97$ \\
Emmetrope $(n=6)$ & $23.51 \pm 0.76$ & $25.26-27.20$ \\
Myope $(n=16)$ & $24.63 \pm 0.87$ & $26.03 \pm 0.61$ \\
High myope $(n=12)$ & & \\
\hline
\end{tabular}

Choroid thickness differed significantly among the three myopic refractive groups $(\mathrm{p}<0.01)$, thickest in emmetropes $(258 \pm 73 \mu \mathrm{m})$ compared to high myopes $(237 \pm 48 \mu \mathrm{m})$. Our data did not show a significant difference between emmetropes and myopic (264 \pm 78 $\mu \mathrm{m})$ groups in the overall measured ChT area $(\mathrm{p}=0.93)$ (Table 2). Eccentricity-dependent differences in ChT were also observed in the emmetropes and myopic refractive groups; however, the high myopic group showed no significant difference in ChT with eccentricity $(\mathrm{p}>0.05$ for all pairwise comparisons). The choroidal thinning associated with high myopia was predominant in the macular regions (fovea, parafovea, and perifovea). Near-periphery and peripheral regions did not show a significantly thin choroid in high myopes. Figure $2 \mathrm{~A}-\mathrm{C}$ illustrates the ChT across all four quadrants as a function of choroidal eccentricity in the three refractive groups.

A significant negative correlation was observed between $\mathrm{ChT}$ and $\mathrm{AL}$ in all measured eccentricities (Pearson's $\mathrm{p}<0.05$ ), where the association between the

Table 2 Overview of Mean Choroidal Thickness Across Measured Regions

\begin{tabular}{|c|c|c|c|c|}
\hline & \multicolumn{4}{|c|}{ Mean \pm SD Choroidal Thickness $(\mu \mathrm{m})$} \\
\hline & All Subjects $(n=34)$ & Emmetropes $(n=6)$ & Myopes $(n=16)$ & High Myopes $(n=12)$ \\
\hline Overall thickness & $257 \pm 81$ & $258 \pm 73$ & $264 \pm 78$ & $237 \pm 48 * t$ \\
\hline \multicolumn{5}{|l|}{ Eccentricity } \\
\hline Fovea $(0.5 \mathrm{~mm})$ & $271 \pm 83$ & $297 \pm 57$ & $286 \pm 72$ & $231 \pm 36 * \dagger$ \\
\hline Parafovea $(1 \mathrm{~mm})$ & $269 \pm 81$ & $287 \pm 62$ & $284 \pm 74$ & $237 \pm 40 * \dagger$ \\
\hline Perifovea $(\mathrm{I} \mathrm{mm})$ & $259 \pm 82$ & $262 \pm 69$ & $272 \pm 81$ & $242 \pm 50^{\dagger}$ \\
\hline Near-periphery $(1.5 \mathrm{~mm})$ & $240 \pm 83$ & $231 \pm 79$ & $251 \pm 84^{*}$ & $237 \pm 61$ \\
\hline Periphery $(2 \mathrm{~mm})$ & $225 \pm 63$ & $210 \pm 71$ & $227 \pm 62$ & $239 \pm 46$ \\
\hline \multicolumn{5}{|l|}{ Quadrant } \\
\hline Superior & $277 \pm 73$ & $273 \pm 62$ & $292 \pm 69$ & $266 \pm 47^{\dagger}$ \\
\hline Temporal & $262 \pm 74$ & $275 \pm 59$ & $263 \pm 72$ & $248 \pm 38$ \\
\hline Inferior & $255 \pm 80$ & $252 \pm 75$ & $276 \pm 78^{*}$ & $238 \pm 37^{\dagger}$ \\
\hline Nasal & $218 \pm 89$ & $231 \pm 93$ & $226 \pm 82$ & $198 \pm 44^{* \dagger}$ \\
\hline
\end{tabular}

Notes: *Significantly thinner choroid in the refractive group compared to the emmetropic group $(p<0.05)$ with adjustment for multiple comparisons (Bonferroni). ${ }^{\dagger}$ Significantly thinner choroid in the high myopic refractive group compared to the myopic group $(\mathrm{p}<0.05)$ with adjustment for multiple comparisons (Bonferroni). 

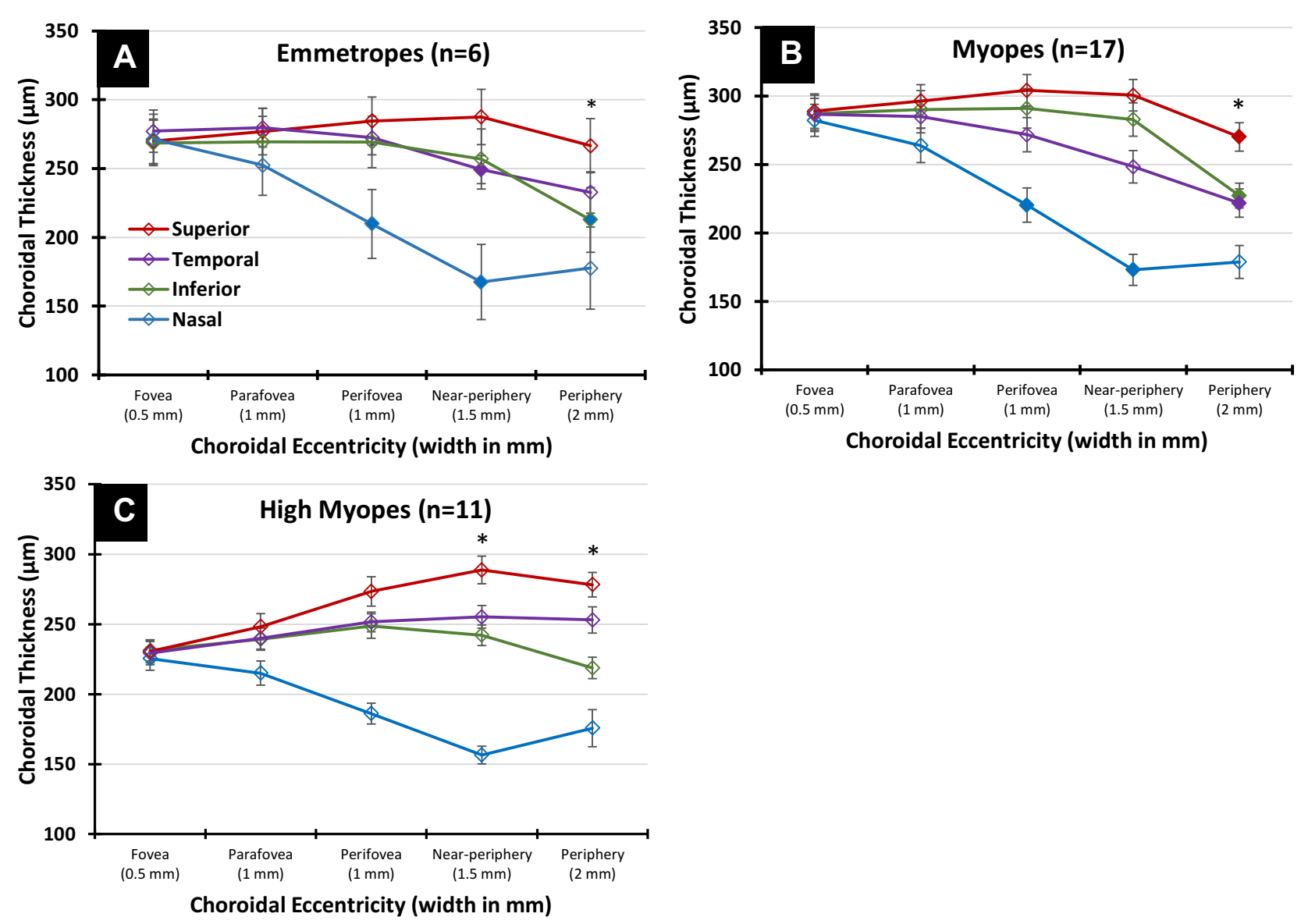

Figure 2 Mean choroidal thickness $(\mu \mathrm{m})$ a across different quadrants as a function of eccentricity in emmetropic (A), myopic (B), and high myopic eyes (C). Error bars represents standard error of the mean. Single asterisks $(*)$ indicate a statistically significant difference $(P<0.05)$ between the superior and the other quadrants. Filled symbols represent a statistically significant difference $(p<0.05)$ in a given choroidal eccentricity compared to the adjacent eccentricity to the left.

two variables lessened with increasing eccentricity. Likewise, a significant negative correlation was also found between $\mathrm{ChT}$ and central spherical equivalent refractive error (Pearson's $\mathrm{p}<0.01$ ). The correlation also weakened with eccentricity.

\section{Diurnal Variation in Wide-Field Choroidal} Thickness and Axial Length

Our data revealed that both ChT and AL underwent significant diurnal variation over time (both $\mathrm{p}<0.05$ ), Figure 3. Diurnal variation amplitude in ChT and AL during the time (from $8 \mathrm{AM}$ to $4 \mathrm{PM}$ ) was computed as the difference between the maximum and minimum values of each subject. Table 3 summarizes the diurnal variation amplitudes means in $\mathrm{ChT}$ and $\mathrm{AL}$ for each refractive group. Means $\pm \mathrm{SD}$ of diurnal variation amplitude in $\mathrm{AL}$ and ChT were $17.8 \pm 11$ and $17.6 \pm 15$, respectively. ChT diurnal variation exhibited a significantly greater amplitude in emmetropic $(21.4 \pm 15 \mu \mathrm{m})$ and myopic $(19 \pm 17 \mu \mathrm{m})$ compared to highly myopic eyes $(14.6 \pm 11 \mu \mathrm{m})$ (main effect: $\left.F_{(2,103)}=4.4, p<0.05\right)$. The reduced amplitude in high myopes was predominant in fovea and parafovea regions, where other eccentric regions did not significantly differ when compared to emmetropic and myopic eyes. Similarly, AL diurnal variation amplitude was significantly larger in emmetropic eyes $(21.3 \pm 8.5 \mu \mathrm{m})$ than in myopic $(18.8 \pm 9.7 \mu \mathrm{m})$ and in highly myopic eyes $(14.5 \pm 12.7$ $\mu \mathrm{m})$ (main effect: $\left.\mathrm{F}_{(2,192)}=14.8, \mathrm{p}<0.001\right)$. No significant difference between right and left eyes was observed in diurnal variation amplitude $(\mathrm{p}>0.05)$.

ChT diurnal variation amplitude data were analyzed $6 \mathrm{~mm}$ from the foveal pit towards the periphery in all four quadrants. Our results showed that ChT diurnal variation amplitude did not differ significantly based on eccentricity (main effect: $\mathrm{F}_{(4,1135)}=0.4, \mathrm{p}=0.79$ ) with amplitude range 18.9 to $17.5 \mu \mathrm{m}$. In comparing the ChT diurnal variation amplitude in all quadrants, our data 


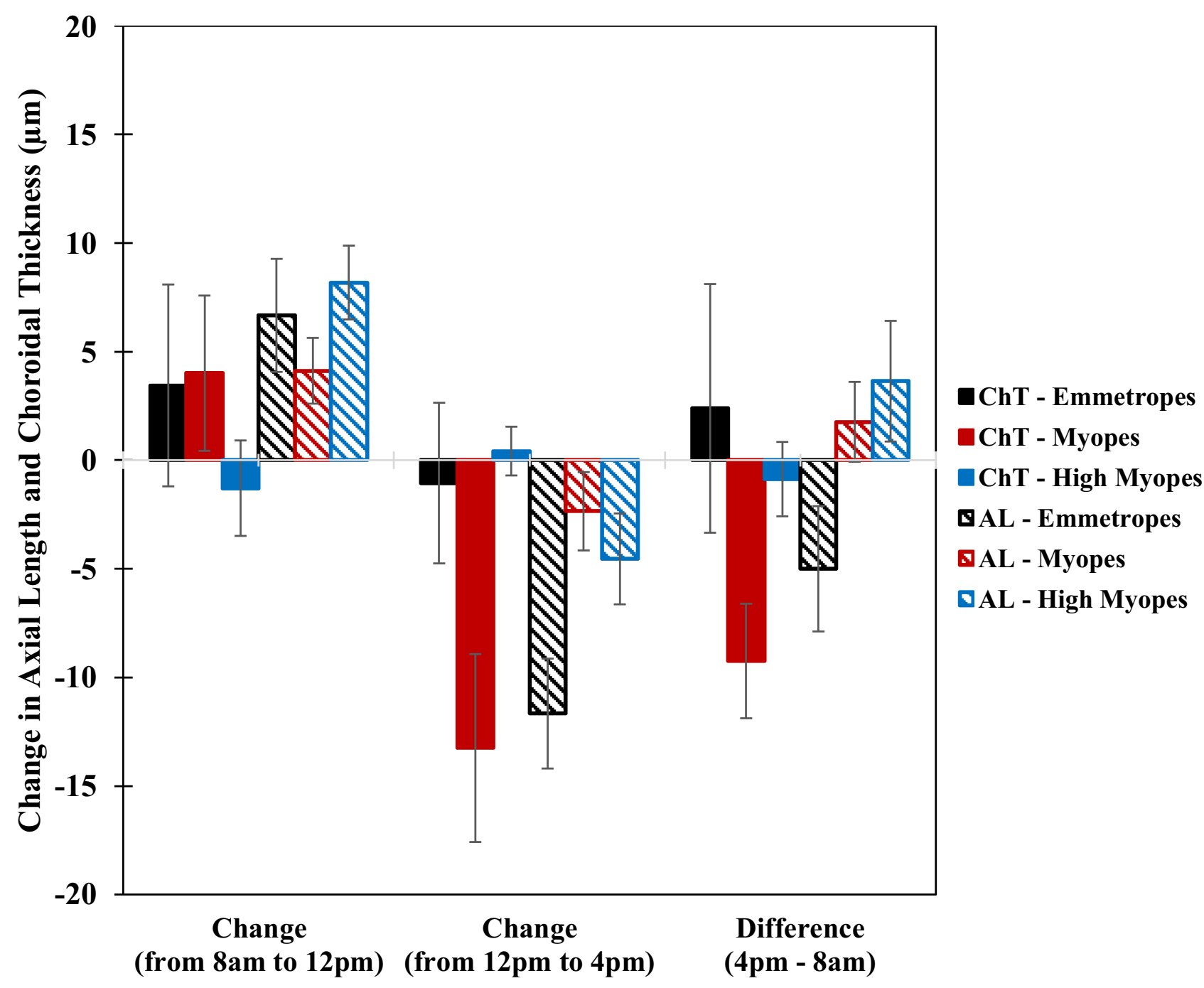

Figure 3 Mean change in subfoveal choroidal thickness $(\mathrm{ChT})$ and axial length $(\mathrm{AL})$ among the three refractive groups. Error bars represent standard error of the mean.

revealed a significant difference (main effect: $\mathrm{F}_{(3,1137)}=$ $15.7, \mathrm{p}<0.001)$ with the nasal $(22.5 \pm 18 \mu \mathrm{m})$ and inferior choroid $(15.5 \pm 12 \mu \mathrm{m})$ exhibited the highest and lowest variation amplitudes, respectively. Pairwise comparisons showed the variation amplitudes are significantly greater in quadrants across the horizontal meridian compared to superior and inferior $(\mathrm{p}<0.05)$, Table 3 .

A significant interaction effect was found for $\mathrm{ChT}$ diurnal variation amplitude between refractive group, quadrant, and eccentricity (refractive group $\times$ quadrant $\times$ eccentricity interaction: $\left.\mathrm{F}_{(33,1135)}=2.2, \mathrm{p}<0.001\right)$.

There was a significant positive correlation between diurnal variation amplitude in subfoveal $\mathrm{ChT}$ and central spherical equivalent refractive error (Pearson's $\mathrm{p}<0.05$ ), where the correlation diminished in the peripheral choroid, Figure 4A. Likewise, there was a statistically significant correlation between diurnal variation amplitude and central spherical equivalent refractive error (Pearson's $\mathrm{p}<$ 0.05), Figure 4B.

\section{Discussion}

Numerous previous studies explored the $\mathrm{ChT}$ in healthy young adults. The majority of studies limited the thickness measurement to the macular region. ${ }^{30-34}$ Recently, several investigated the normal ChT distribution over a wide field. ${ }^{13,14,16}$ HoseiniYazdi et al investigated the variation of ChT over the central $14 \mathrm{~mm}\left(55^{\circ}\right)$ area centered on the fovea in 27 young adults with varying refractive conditions. ${ }^{14}$ Rasheed et al also studied the mid-equatorial ChT. In this study, we observed the variation in $\mathrm{ChT}$ in the young adult population over the central $12 \mathrm{~mm}$ in both vertical and horizontal meridians $\left(47^{\circ} \times 47^{\circ}\right)$. The choroid exhibited a significant eccentricity-dependent 
Table 3 Overview of the Diurnal Variation Amplitude in Axial Length and Choroidal Thickness in the Three Myopic Refractive Groups

\begin{tabular}{|c|c|c|c|c|}
\hline & \multicolumn{4}{|c|}{ Mean \pm SD Diurnal Variation Amplitude $(\mu \mathrm{m})$} \\
\hline & All Subjects $(n=34)$ & Emmetropes $(n=6)$ & Myopes $(n=16)$ & High Myopes $(n=12)$ \\
\hline Axial length & $17.8 \pm 11$ & $21.3 \pm 8.5$ & $18.8 \pm 9.7$ & $14.5 \pm 12.7^{* \dagger}$ \\
\hline Choroidal thickness (ChT) & $17.6 \pm 15$ & $21.4 \pm 15$ & $19.0 \pm 17$ & $14.6 \pm 11^{* t}$ \\
\hline \multicolumn{5}{|l|}{ ChT: Eccentricity } \\
\hline Fovea $(0.5 \mathrm{~mm})$ & $18.5 \pm 15$ & $23 \pm 15$ & $19 \pm 17$ & $13 \pm 10 * t$ \\
\hline Parafovea $(1 \mathrm{~mm})$ & $18.8 \pm 15$ & $24 \pm 15$ & $20 \pm 17$ & $13 \pm 8^{* \dagger}$ \\
\hline Perifovea $(\mathrm{I} \mathrm{mm})$ & $18.9 \pm 14$ & $23 \pm 16$ & $18 \pm 14$ & $15 \pm 12$ \\
\hline Near-periphery $(1.5 \mathrm{~mm})$ & $17.5 \pm 15$ & $18 \pm 14$ & $18 \pm 16$ & $16 \pm 13$ \\
\hline Periphery $(2 \mathrm{~mm})$ & $17.7 \pm 16$ & $18 \pm 13$ & $20 \pm 17$ & $16 \pm 13$ \\
\hline \multicolumn{5}{|l|}{ ChT: Quadrant } \\
\hline Superior & $16.0 \pm 12$ & $19.9 \pm 13$ & $16.5 \pm 13$ & $11.6 \pm 8^{*}$ \\
\hline Temporal & $20.2 \pm 17$ & $22.5 \pm 13$ & $22.3 \pm 19$ & $15.8 \pm 12^{* t}$ \\
\hline Inferior & $15.5 \pm 12$ & $18.9 \pm 15$ & $14.7 \pm 12$ & $12.8 \pm 8$ \\
\hline Nasal & $22.5 \pm 18$ & $25.1 \pm 18$ & $23.1 \pm 19$ & $19.2 \pm 15$ \\
\hline
\end{tabular}

Notes: *Significantly less diurnal variation amplitude in the refractive group compared to the emmetropic group ( $\mathrm{p}<0.05$ ) with adjustment for multiple comparisons (Bonferroni). 'Significantly less diurnal variation amplitude in the high myopic refractive group compared to the myopic group ( $\mathrm{p}<0.05)$ with adjustment for multiple comparisons (Bonferroni).

thickness variation, with progressive thinning observed beyond the parafovea $(259 \pm 82 \mu \mathrm{m})$ into the periphery $(225$ $\pm 63 \mu \mathrm{m})$. In addition, ChT varied based on the quadrant, with superior $(277 \pm 73 \mu \mathrm{m})$ choroid exhibited the thickest and nasal $(218 \pm 89 \mu \mathrm{m})$ exhibiting the thinnest. Superior ChT did not show an eccentricity-dependent change in thickness, while the nasal choroid thinned markedly towards the periphery (summarized graphically in Figure 2A-C). Our findings were consistent with those reported in Hoseini-Yazdi's and Rasheed's studies in the ChT morphological distribution. Previous studies indicated that the diameter of choroidal vessels and the ratio between horizontal and vertical choroidal veins diameter were significantly correlated with $\mathrm{ChT}{ }^{13,35}$ Another study found that eyes had asymmetry in the distribution of choroidal veins, with superior-temporal choroid as the preferential choroidal venous drainage route. ${ }^{36}$ The spatial location of the choroidal veins most likely explained the observed ChT variation in different quadrants. Previous studies reported differences in the density of choroidal microvascular and large vascular components, with high density in the central fovea and reduced density toward the peripheral regions. ${ }^{37-40}$ The eccentricity-dependent variations in choroidal vascular density are likely the main contributing factor to the observed morphological variation in ChT.

The ChT averaged across the entire measured regions was $9 \%$ thinner in highly myopic eyes $(237 \pm 48 \mu \mathrm{m})$ compared to emmetropes $(258 \pm 73 \mu \mathrm{m})$, which was consistent with previously reported in healthy adults ${ }^{31,34}$ and children. ${ }^{27}$ Previous studies also reported a significant correlation between $\mathrm{ChT}$ and $\mathrm{AL}$ or refractive error across the macular region. ${ }^{14,30-32}$ Hoseini-Yazdi reported a significant correlation between macular ChT and AL or refractive error with no significant correlation observed beyond the perifoveal area (outside 5-mm central area). Our data showed a significant association in ChT with both $\mathrm{AL}$ and refractive error in all measured regions, including macular and peripheral regions (beyond 5-mm central region) ( $\mathrm{P}<0.05$, Pearson's correlation coefficient), for which the correlation reduced towards the periphery. The observed correlation in the peripheral regions might be explained by the a wide range of myopic refractive error $(-0.50$ to $-10.50 \mathrm{D})$ in our sample. The choroidal thinning associated with myopia was also reported in children; however, the magnitude of foveal choroidal thinning seen in the pediatric population $(10-16 \%)^{27,41}$ is less than found in adults $(20-26 \%),{ }^{14}$ consistent with what was found in highly myopic eyes in comparison to emmetropic eyes $(22 \%)$ in the subfoveal region.

The present study was also designed to determine the diurnal variation amplitude in $\mathrm{ChT}$ and $\mathrm{AL}$ among a range of myopic refractive errors. Our data showed that, on average, the choroid was thicker at 8:00 AM, thinnest at 

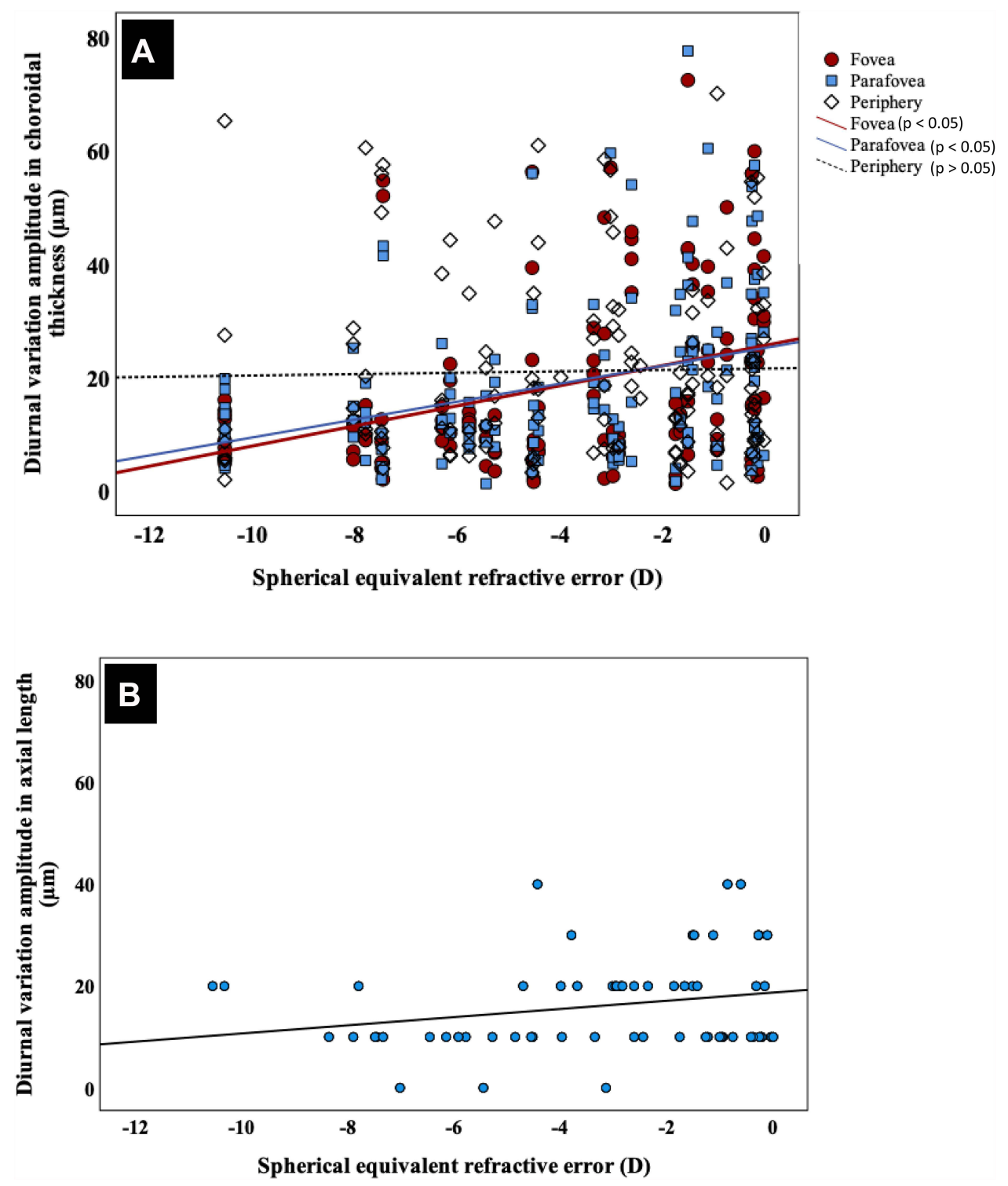

Figure 4 Scatter plots graphs illustrating the association between the diurnal variation amplitude in choroidal thickness and refraction (A) and between the variation in axial length and refraction $(\mathbf{B})$.

12:00 PM, then progressively increased in thickness at 4:00 PM, which was consistent with previously published studies. ${ }^{5}$ As expected, the diurnal variation pattern in $\mathrm{AL}$ was in antiphase to ChT.

With regards to the diurnal variation amplitude in ChT as a function of location, eccentricity, and level of myopia, our findings indicated that high myopes experienced a statistically significant reduced diurnal variation amplitude in ChT predominantly in foveal and parafoveal regions compared to emmetropic and myopic eyes. Beyond the parafoveal region to the peripheral region, the diurnal variation amplitude in ChT was not significantly different between the three refractive groups. Due to the spatial anatomical vascular and contractile non-vascular cells arrangement of the choroid, it is likely that the significant choroidal thinning associated with higher myopia may limit the choroidal tissue from changing its thickness at a greater amplitude throughout the day as observed in emmetropic eyes. The findings of significantly reduced variation amplitude in high myopes were limited in the central choroid (fovea and parafovea), which may suggest that the central choroid had a greater contribution to active choroidal changes related to myopia development compared to the peripheral region.

In previous studies investigating the diurnal variation in ChT, Gabriel et al assessed the variation amplitude in 
ChT across $36^{\circ} \times 36^{\circ}$ field using an SD-OCT in 19 healthy individuals. $^{42}$ In our investigation with similar age and AL distribution of the subjects, the mean diurnal ChT variation for all regions was $17.6 \pm 15 \mu \mathrm{m}$, comparable to that reported by Gabriel et al. As the ChT was quadrant- and eccentricity-dependent, it was not surprising that the diurnal variation amplitude in ChT was also not equally distributed in the measured choroidal regions, with temporal and nasal choroid showed the most variation amplitude. Similarly, Gabriel et al reported a significant diurnal variation in all quadrants except in the superior choroid. ${ }^{42}$

The overall percentage of ChT diurnal variation was also similar to previously reported by Tan et al and Usui et al, 4,5 emmetropic eyes demonstrated the highest percentage change, followed by myopic and highly myopic eyes. It has been suggested that the diurnal variation that is observed in ChT may influence ocular growth and myopia development. Chicks raised in constant light or total darkness (eliminating diurnal cues) had excessive ocular growth and myopia development. Related, recent growing evidence from clinical studies suggested a protective effect of outdoor time on myopia development. These combined findings supported the plausibility of the importance of maintaining a normal physiologic diurnal variation for normal ocular growth. In addition, ChT response to induced myopic and hyperopic optical defocus was observed in both animals and humans. Recent studies on chicks $^{17}$ and humans ${ }^{18}$ have shown the ChT response to optical defocus depends on the time of the day, which further suggests a potential interaction between the choroidal diurnal variation and its defocus-induced changes as a modulator for ocular growth.

Anatomically, the choroid serves as the interlayer between the retina and the sclera, which not only provides oxygen and nutrients to the adjacent structures but also relays defocus signals originated from the sensory retina, through RPE, to the sclera. The quadrant- and eccentricitydependent distribution of ChT strongly supports a relatively independent regional modulation of the choroid and its role in relaying the defocus signals. It has been reported that the choriocapillaris plexus function as relative independent "lobules" and receive segmental arterial feeding and form segregated venous drainage. ${ }^{43}$ This relative separation of choroidal vascular formation hence its potential functional independence, has critical clinical relevance in myopes. First of all, as the relay of the defocus signals, localized change in ChT and blood flow may serve an essential role in the localized ocular growth reported in various myopia animal models. Consequently, the early quantification of these localized choroidal changes could potentially increase the sensitivity and reduce the latency in detecting the pre-clinical stage of accelerated axial growth and excessive scleral stretching, hence preventing the onset of myopia. Secondly, a better understanding of the quadrant- and the eccentricity-dependent response of choroid to the myopia-genic and its controlling signals allows for a more individualized approach in the designs of anti-myopia treatment, in which the dosage to different regions of the retinal field could be custom-tailored based on the specific choroidal profile of each patient. Finally, since the progressive thinning of the choroid is an important intermediate step of the pathogenesis of myopic maculopathy, a thorough evaluation of the localized choroidal changes may facilitate more targeted drug delivery for better efficacy and safety of the treatment.

To the best of our knowledge, this was the first study to assess the diurnal variation over a larger field of the choroid and across a wide range of myopic refractive error. However, several limitations to this study need to be acknowledged. First of all, the majority of subjects in this study were females, which might have a confounding effect on the overall results due to the physiological differences between males and females in their diurnal cycles. However, the impact from this potential confounding is unlikely to be significant as the gender distribution was not significantly different in all refractive groups. Nonetheless, the external generalization of the results may be limited due to the gender imbalance. Secondly, the ChT variation was not assessed at night hence unable to provide a detailed diurnal fluctuation of $\mathrm{ChT}$ and $\mathrm{AL}$ beyond the measured time points, and instead data extrapolation was utilized, which may limit its applicability to the true pattern at night. Thirdly, despite the attempt to control major known confounders to diurnal changes such as alcoholic and caffeinated beverages consumption, other physiologic factors such as hydration were not assessed and controlled. Finally, the inter-examiner reliability on OCT image analysis was measured in a partially subjective manner, which could be improved by utilizing more quantitative or objective methods in future studies.

\section{Conclusion}

ChT distribution followed a quadrant- and eccentricitydependent pattern, which the superior choroid being thickest, and the nasal quadrant being thinnest. ChT was 
significantly and negatively associated with the level of myopia. High myopes had significant choroidal thinning across the foveal, parafoveal, and perifoveal regions and less prominent diurnal variation in $\mathrm{ChT}$ and $\mathrm{AL}$ compared to emmetropic and lower myopic eyes.

\section{Acknowledgments}

We wish to thank the team of the Contact Lens and Visual Optics Laboratory, Queensland University of Technology, Australia, for sharing the technological support for measuring choroidal thickness. Special thanks to Dr. Adam Preston for his assistant in data preparation and analysis.

This work was presented at the American Academy of Optometry on Oct 10, 2020, as a poster.

\section{Author Contributions}

All authors made a significant contribution to the work reported, whether that is in the conception, study design, execution, acquisition of data, analysis and interpretation, or in all these areas; took part in drafting, revising or critically reviewing the article; gave final approval of the version to be published; have agreed on the journal to which the article has been submitted; and agreed to be accountable for all aspects of the work.

\section{Funding}

There is no funding to report for this submission.

\section{Disclosure}

The authors report no conflicts of interest for this work and wish to confirm that there are no known conflicts of interest associated with this publication. There has been no significant financial support for this work that could have influenced its outcome.

\section{References}

1. Zee PC, Attarian H, Videnovic A. Circadian rhythm abnormalities. Contin Lifelong Learn Neurol. 2013;19(1):132. doi:10.1212/01. CON.0000427209.21177.aa

2. Chakraborty R, Read SA, Collins MJ. Diurnal variations in axial length, choroidal thickness, intraocular pressure, and ocular biometrics. Investig Opthalmol Vis Sci. 2011;52(8):5121. doi:10.1167/ iovs.11-7364

3. Nickla DL, Wildsoet C, Wallman J. Visual influences on diurnal rhythms in ocular length and choroidal thickness in chick eyes. Exp Eye Res. 1998;66(2):163-181.

4. Tan CS, Ouyang Y, Ruiz H, Sadda SR. Diurnal variation of choroidal thickness in normal, healthy subjects measured by spectral domain optical coherence tomography. Investig Opthalmol Vis Sci. 2012;53 (1):261. doi:10.1167/iovs.11-8782.
5. Usui S, Ikuno Y, Akiba M, et al. Circadian changes in subfoveal choroidal thickness and the relationship with circulatory factors in healthy subjects. Invest Ophthalmol Vis Sci. 2012;53(4):2300-2307. doi:10.1167/iovs.11-8383

6. Maruko I, Iida T, Sugano Y, Ojima A, Sekiryu T. Subfoveal choroidal thickness in fellow eyes of patients with central serous chorioretinopathy. Retina. 2011;31(8):1603-1608.

7. Fong AH, Li KK, Wong D. Choroidal evaluation using enhanced depth imaging spectral-domain optical coherence tomography in Vogt-Koyanagi-Harada disease. Retina. 2011;31(3):502-509.

8. Ikuno Y, Tano Y. Retinal and choroidal biometry in highly myopic eyes with spectral-domain optical coherence tomography. Investig Opthalmol Vis Sci. 2009;50(8):3876. doi:10.1167/iovs.08-3325

9. Chung SE, Kang SW, Lee JH, Kim YT. Choroidal thickness in polypoidal choroidal vasculopathy and exudative age-related macular degeneration. Ophthalmology. 2011;118(5):840-845.

10. Usui S, Ikuno Y, Miki A, Matsushita K, Yasuno Y, Nishida K. Evaluation of the choroidal thickness using high-penetration optical coherence tomography with long wavelength in highly myopic normal-tension glaucoma. Am J Ophthalmol. 2012;153(1):10-16.e1.

11. Esmaeelpour M, Považay B, Hermann B, et al. Mapping choroidal and retinal thickness variation in type 2 diabetes using three-dimensional 1060-nm optical coherence tomography. Invest Ophthalmol Vis Sci. 2011;52(8):5311-5316.

12. Nickla DL, Wallman J. The multifunctional choroid. Prog Retin Eye Res. 2010;29(2):144-168.

13. Rasheed MA, Singh SR, Invernizzi A, et al. Wide-field choroidal thickness profile in healthy eyes. Sci Rep. 2018;8(1):17166.

14. Hoseini-Yazdi H, Vincent SJ, Collins MJ, Read SA, Alonso-Caneiro D. Wide-field choroidal thickness in myopes and emmetropes. Sci Rep. 2019;9(1):1. doi:10.1038/s41598-019-39653-w

15. Mohler KJ, Draxinger W, Klein T, et al. Combined $60^{\circ}$ wide-field choroidal thickness maps and high-definition en face vasculature visualization using swept-source megahertz OCT at $1050 \mathrm{~nm}$. Investig Opthalmol Vis Sci. 2015;56(11):6284. doi:10.1167/iovs.15-16670

16. Breher K, Terry L, Bower T, Wahl S. Choroidal Biomarkers: a repeatability and topographical comparison of choroidal thickness and choroidal vascularity index in healthy eyes. Transl Vis Sci Technol. 2020;9(11):8. doi:10.1167/tvst.9.11.8.

17. Nickla DL, Jordan K, Yang J, Totonelly K. Brief hyperopic defocus or form deprivation have varying effects on eye growth and ocular rhythms depending on the time-of-day of exposure. Exp Eye Res. 2017;161:132-142. doi:10.1016/j.exer.2017.06.003

18. Moderiano D, Do M, Hobbs S, et al. Influence of the time of day on axial length and choroidal thickness changes to hyperopic and myopic defocus in human eyes. Exp Eye Res. 2019;182:125-136. doi:10.1016/j.exer.2019.03.019

19. Deng J, Li X, Jin J, et al. Distribution pattern of choroidal thickness at the posterior pole in Chinese children with myopia. Invest Ophthalmol Vis Sci. 2018;59(3):1577-1586. doi:10.1167/iovs.17-22748

20. Kang HM, Woo YJ, Koh HJ, Lee CS, Lee SC. The effect of consumption of ethanol on subfoveal choroidal thickness in acute phase. Br J Ophthalmol. 2016;100(3):383-388.

21. Zengin MO, Cinar E, Karahan E, Tuncer I, Kucukerdonmez C. The effect of caffeine on choroidal thickness in young healthy subjects. Cutan Ocul Toxicol. 2015;34(2):112-116.

22. Ulaş F, Çelik F, Doğan Ü, Çelebi S. Effect of smoking on choroidal thickness in healthy smokers. Curr Eye Res. 2014;39(5):504-511.

23. Girard MJA, Strouthidis NG, Ethier CR, Mari JM. Shadow removal and contrast enhancement in optical coherence tomography images of the human optic nerve head. Investig Opthalmol Vis Sci. 2011;52 (10):7738. doi:10.1167/iovs.10-6925

24. Read SA, Collins MJ. The short-term influence of exercise on axial length and intraocular pressure. Eye. 2011;25(6):767-774. 
25. Woodman EC, Read SA, Collins MJ, Axial length and choroidal thickness changes accompanying prolonged accommodation in myopes and emmetropes. Vision Res. 2012;72:34-41.

26. Alonso-Caneiro D, Read SA, Vincent SJ, Collins MJ, Wojtkowski M. Tissue thickness calculation in ocular optical coherence tomography. Biomed Opt Express. 2016;7(2):629. doi:10.1364/BOE.7.000629.

27. Read SA, Collins MJ, Vincent SJ, Alonso-Caneiro D. Choroidal thickness in myopic and nonmyopic children assessed with enhanced depth imaging optical coherence tomography. Invest Ophthalmol Vis Sci. 2013;54(12):7578-7586.

28. Alonso-Caneiro D, Read SA, Collins MJ. Automatic segmentation of choroidal thickness in optical coherence tomography. Biomed $O p t$ Express. 2013;4(12):2795.

29. Read SA, Collins MJ, Vincent SJ, Alonso-Caneiro D. Macular retinal layer thickness in childhood. Retina. 2015;35(6):1223-1233.

30. Agawa T, Miura M, Ikuno Y, et al. Choroidal thickness measurement in healthy Japanese subjects by three-dimensional high-penetration optical coherence tomography. Graefes Arch Clin Exp Ophthalmol. 2011;249(10):1485-1492.

31. Hirata M, Tsujikawa A, Matsumoto A, et al. Macular choroidal thickness and volume in normal subjects measured by swept-source optical coherence tomography. Investig Opthalmol Vis Sci. 2011;52 (8):4971. doi:10.1167/iovs.11-7729

32. Ouyang Y, Heussen FM, Mokwa N, et al. Spatial distribution of posterior pole choroidal thickness by spectral domain optical coherence tomography. Investig Opthalmol Vis Sci. 2011;52(9):7019. doi:10.1167/iovs.11-8046

33. Sanchez-Cano A, Orduna E, Segura F, et al. Choroidal thickness and volume in healthy young white adults and the relationships between them and axial length, ammetropy and sex. Am J Ophthalmol. 2014;158(3):574-583.e1.
34. Tan C, Cheong KX, Lim LW, Li KZ. Topographic variation of choroidal and retinal thicknesses at the macula in healthy adults. $\mathrm{Br}$ J Ophthalmol. 2013;98(3):339-344.

35. Tanabe $\mathrm{H}$, Ito $\mathrm{Y}$, Iguchi $\mathrm{Y}$, Ozawa $\mathrm{S}$, Ishikawa $\mathrm{K}$, Terasaki $\mathrm{H}$. Correlation between cross-sectional shape of choroidal veins and choroidal thickness. Jpn J Ophthalmol. 2011;55(6):614-619.

36. Mori K. Asymmetry of choroidal venous vascular patterns in the human eye. Ophthalmology. 2004;111(3):507-512.

37. McLeod DS, Lutty GA. High-resolution histologic analysis of the human choroidal vasculature. Invest Ophthalmol Vis Sci. 1994;13:3799-3811.

38. Esmaeelpour M, Kajic V, Zabihian B, et al. Choroidal haller's and sattler's layer thickness measurement using 3-dimensional 1060-nm optical coherence tomography. PLoS One. 2014:9(6); e99690.

39. Adhi M, Ferrara D, Mullins RF, et al. Characterization of choroidal layers in normal aging eyes using enface swept-source optical coherence tomography. PLoS One. 2015;10(7):e0133080.

40. Ferrara D, Waheed NK, Duker JS. Investigating the choriocapillaris and choroidal vasculature with new optical coherence tomography technologies. Prog Retin Eye Res. 2016;52:130-155. doi:10.1016/j. preteyeres.2015.10.002

41. Jin P, Zou H, Zhu J, et al. Choroidal and retinal thickness in children with different refractive status measured by swept-source optical coherence tomography. Am J Ophthalmol. 2016; 168;164-176. doi: 10.1016/j.ajo.2016.05.008

42. Gabriel M, Esmaeelpour M, Shams-Mafi F, et al. Mapping diurnal changes in choroidal, haller's and sattler's layer thickness using 3dimensional 1060-nm optical coherence tomography. Graefes Arch Clin Exp Ophthalmol. 2017;255(10):1957-1963.

43. Spaide RF, Ledesma-Gil G, Gemmy Cheung CM. Intervortex venous anastomosis in pachychoroid-related disorders. Retina. 2021;41 (5):997-1004.
Clinical Ophthalmology

\section{Publish your work in this journal}

Clinical Ophthalmology is an international, peer-reviewed journal covering all subspecialties within ophthalmology. Key topics include: Optometry; Visual science; Pharmacology and drug therapy in eye diseases; Basic Sciences; Primary and Secondary eye care; Patient Safety and Quality of Care Improvements. This journal is indexed on PubMed

\section{Dovepress}

Central and CAS, and is the official journal of The Society of Clinical Ophthalmology (SCO). The manuscript management system is completely online and includes a very quick and fair peer-review system, which is all easy to use. Visit http://www.dovepress.com/ testimonials.php to read real quotes from published authors. 